\title{
P-1065 - Diabetes Training for Paramedical Personnel in India - National Capacity Building for Management of Diabetes
}

\author{
Vinitha Ramachandran, Shobhana Ramachandran, Narayanasamy Murugesan, \\ Chamukuttan Snehalatha, Arun Nanditha, Arun Raghavan, Ambady Ramachandran
}

\author{
(IDRF \\ India Diabetes Research Foundation \& Dr. A. Ramachandran's Diabetes Hospitals \\ Chennai, India.
}

Background: A 3 year programme to train doctors and paramedical personnel from 10 states of India was conducted by India Diabetes Research Foundation (IDRF), Chennai, India, which is a nodal centre for facilitating the national programme for early diagnosis, optimal management and prevention of diabetes and other non-communicable diseases.

Aims: A short structured 3 day training programme was conducted for paramedical personnel at the IDRF. The immediate impact of the training was assessed using a structured questionnaire. This paper shows the immediate impact of the paramedical training.

Methods: In this programme 4327 (Men: Women, 1439: 2888) paramedical personnel were trained in 3 day diabetes workshops. Majority (64\%) were from semi urban or rural areas. The analysis of the immediate impact of the training on the knowledge and attitude of the participants were analysed using pre and posttraining knowledge score, feedback on usefulness of the training modules and on proposed activities to improve their practice.

Figure

Number of paramedical personnel from 10 states of India

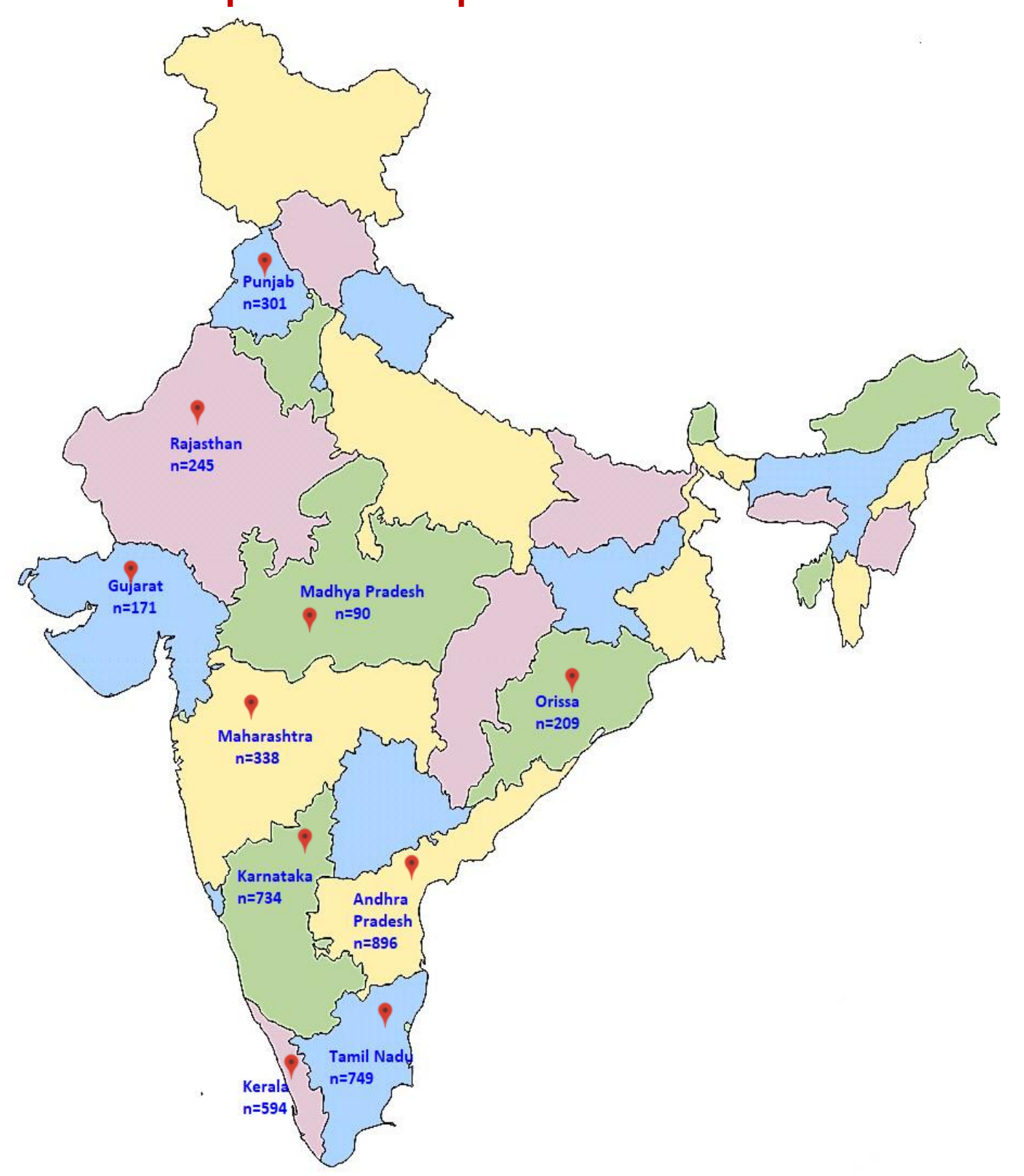

Results: Significant improvements in knowledge scores on general aspects on diabetes, its treatment and complications, were seen in all categories of age, qualification, institutional background and geographical areas. The table shows pretraining and post-training scores in the total group.

Table

Distribution of knowledge scores at pre-training and post-training assessments

\begin{tabular}{|c|c|c|c|c|c|c|}
\hline Modules & \multicolumn{2}{|l|}{ General } & \multicolumn{2}{|c|}{ Treatment } & \multicolumn{2}{|c|}{ Complications } \\
\hline \multirow[t]{3}{*}{$\begin{array}{l}\text { Maximum } \\
\text { Scores }\end{array}$} & 17 & & 21 & & 6 & \\
\hline & \multicolumn{6}{|c|}{ Scores- Median values (Inter-quartile range) } \\
\hline & Pre-test & Post-test* & Pre-test & Post-test* & Pre-test & Post-test* \\
\hline Total $(n=4327)$ & $10(7-12)$ & $13(11-14)$ & $9(7-12)$ & $13(10-15)$ & $4(1-5)$ & $6(5-6)$ \\
\hline Male $(n=1439)$ & $10(7-12)$ & $13(11-14)$ & $9(6-11)$ & $12(10-15)$ & $5(1-5)$ & $6(5-6)$ \\
\hline Female $(n=2888)$ & $10(7-12)$ & $13(11-14)$ & $10(7-12)$ & $13(10-15)$ & $4(1-5)$ & $6(5-6)$ \\
\hline
\end{tabular}

* Pre-test Vs Post-test p<0.0001

Improvement occurred even among the rural participants who had low pre-training scores. There was no correlation between age and the knowledge scores. Many of the trainees implemented the knowledge gained for improving the practice profile in their respective work places.

Discussion: Short-term training of paramedical personnel was effective in improving basic knowledge on diabetes among all categories of participants and was able to create keen interest among them to improve practice in their local areas. India has shown that health workers can be trained effectively on a national scale by the concerted efforts of the government in collaboration with educational and training bodies ${ }^{1,2}$. This paper provides evidence that short term training can be effective in improving basic knowledge on diabetes among all categories of paramedical health workers and arouse keen interest among them to improve practices for diabetes care delivery in their areas and create a cascading effect by training their colleagues and work as a team. The long term effect on diabetes care delivery has to be assessed over time but will depend on several other health system factors such as their continued involvement with diabetes care delivery, management support, continued availability of resources and facility for diagnosis, monitoring, treatment and education for diabetes. These factors are as important as availability of trained staff to improve long term health care outcomes. 